\title{
A Contrast Measure based Approach to Binaries Handwritten Documents through MRF
}

\author{
Bharti Bansinge \\ M.Tech Scholar \\ Department of Computer Science and Engineering \\ Maulana Azad National Institute of Technology \\ Bhopal, 462051, India
}

\author{
R.K. Pateriya \\ Associate Professor \\ Department of Computer Science and Engineering \\ Maulana Azad National Institute of Technology \\ Bhopal, 462051, India
}

\begin{abstract}
Document binarization plays important role to preserve the historical document. Recently number of researcher present numerous techniques of document binarization that can vary in sensitivity, quality and some more control parameters. The document image binarization focuses on extracting the text and background of the image. In doing this the edge detection approach also played the crucial role. In this paper a framework for digitations of historical physical document has been proposed. This framework suggest to use Markov random function to evaluate contrast of pixel and try to overcome the problem of appearance of a single document that can vary greatly depending on factors such as lighting, viewing angle. Following that, proposed framework uses this energy to differentiate foreground and background ink. Final binaries image document have significant enhance in PSNR (db) value. Proposed scheme use DIBCO (2013) for evaluation and validation.
\end{abstract}

\section{Keywords}

Document digitization, Markov Random field, Contrast measurement, Gaussian filter, Weiner filter

\section{INTRODUCTION}

Document image analysis (DIA) intends to extract the textual information from image document. Document image binarization, as a pre-processing step of many DIA systems, is very important for the document image understanding and has been investigated for decades. Document digitization is an elderly but still a difficult task [1]. Appearance of historical handwritten document varies with quality of ink and periodic shadings that leads to degrade quality of image. Quality of binarization may also vary within single document with light and view angle of different portion of physical document.

Recently numerous of image binarization techniques have been proposed and achieved high milestone. But researcher still try to binaries, degraded document images with satisfactory level and needs further study. The major challenge lies with the modeling of the document foreground/background that often suffers from various types of document degradations such as uneven illumination, image contrast variation, bleed-through, and smear.

Digitations of physical documents are usually use a representation of 24-bit color, or may be 8 bits of grayscale [2]. In most of recent application, these representation techniques do not include all of the data available in original physical document, but retaining more than enough. Recently research leads to retain a single bit per pixel document. Loyalty of digitations of physical document is relatively low, since information is loss, which is very much related to the primary content of the document. Many documents are produced using a monochrome ink for writing, and their meanings are incorporated exclusively for distribution of ink, a bit pattern representing the document explicitly.

Of course, the deduction of correct digitations of a document from color or grayscale representation can be difficult. The physical deterioration of the document, image illumination conditions and limits of the unfavorable resolution can contribute to obscure the original pattern. Now these days many researchers proposed numerous algorithms for digitations of physical document towards this dispute. In fact standard document image binarization contest (DIBCO-13) held in-order to gather an deep researcher [3]. However, the results of these competitions prove that there is always room for enhancement in the quality of automatic binarization.

\section{RELATED WORK}

Bolan Su [4] proposed a document image binarization framework that makes use of the Markov Random Field model. This framework divides the document image pixels into three categories using established binarization method. The uncertain pixels are then classified using those pixels that belong to foreground and background categories. Subsequently Bolan Su [5] also presents an adaptive image contrast based document image binarization technique that is tolerant to different types of document degradation such as uneven illumination and document smear. Their technique is simple and robust, only few parameters are involved. Moreover, it works for different kinds of degraded document images. Their technique makes use of the local image contrast that is evaluated based on the local maximum and minimum.

David Hebert [6] proposed a framework for combining multiple experts dedicated to the binarization of document images. This framework is based on a discrete conditional random field model which allows training to optimize the final binarization decision at pixel level. This system has the advantage of introducing contextual combination rules of the individual expert decisions. The approach is evaluated for document image binarization by combining standard binarization algorithms.

Karthika M [7] proposes a method to perform document image binarization using bit-plane slicing. Their method is deployed, based on the rule of divide-and-conquer. As per this technique, the 8 bit planes of the gray image are extracted and processed separately, at the end of which, the results are combined to give the final binarized output

Apart from above in 2014 Otsu's method for document digitization is an parameter less global threshold method .In this method presence of separate distributions for background and text has been assume, and calculates a threshold value in such a means that lead to minimize the difference between 
two distributions [8]. The limit for the distribution of two Otsu method was eliminated in [8], where the modes of degradation in the image histogram does one remove one applying recursively Otsu's method until only one mode remains in the picture. In other work, the overall limitation of the method is removed [9] and an adaptation method is introduced, which uses the same concept as the Otsu method, but local patches.

B. Gatos [10] introducing a binarization method in which initially obtains a coarse binarization of the document image which is forwarded by a rough background estimation. In the next step, local threshold values are calculated based on the estimated background and some parameters. These threshold values are used to calculate the final binarization that is postprocessed to remove noise.

B. Gatos, K. Ntirogiannis and I. Pratikakis [11] presented their method in DIBCO'09. This comprises four steps. First removing the background by polynomial fit of the lines. Second detecting the contours of the race using Otsu's method on the gradient information, then local threshold by averaging the detected pixels in a local neighborhood window edges, and finally post-processing of results.

Fabrizio and Marcotegui [12] introduced a method in DIBCO'9 based on morphological mapping operator shaft rocker [13]. To avoid salt and pepper noise associated with balancing mapping, are excluded from the analysis pixel erosion and dilation which are too close. Pixels are then classified as text, background pixels and uncertain. The uncertain pixels are assigned to the text and background, depending on their class boundary.

Although images of the documents may suffer from degradation but we can assume that there are areas that could be described as actual text or background to enhance the quality of an image. This hypothesis has been the basis of many learning methods, which are based on a rough estimate of the text boxes and background and then try to learn their behavior to classify regions found in the confusion range [13 14].In [15], a frame using a binarization method is presented to identify three classes; i.e. text, background, and uncertain pixels, the pixels of uncertainty reclassified using a classifier trained using the classes of the text and background.

\section{PROPOSED MODEL}

Proposed framework for handwritten historical document binarization is show in figure 2. Proposed framework consist color to gray conversion, contrast measurement, pixel segmentation based back ground elimination and refine foreground image by filter out rough foreground image.

Grayscale Conversion: - Proposed framework initially applies grayscale conversion [b8] for eliminating noisy areas, smoothing of background texture as well as contrast enhancement between background and text areas as show in figure.

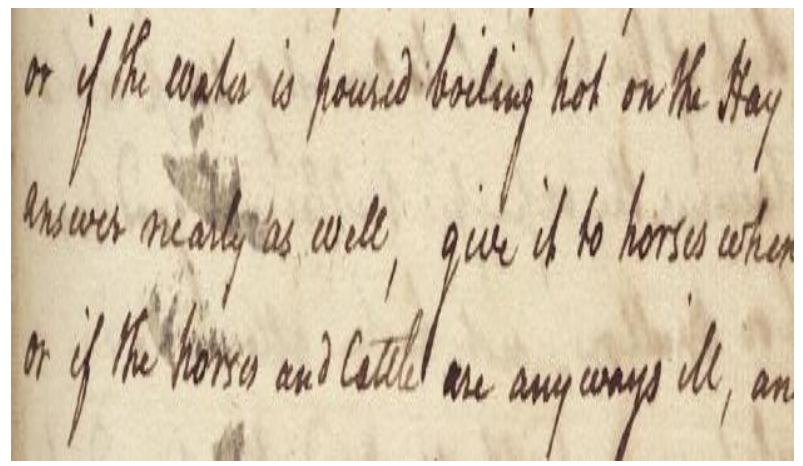

Fig 1: Input handwritten image

Contrast Measure: Subsequently proposed frame apply Markov random field for contrast measure of a pixel against its background. And statically calculate threshold that leads to segment out pixel into three category document background pixels, document foreground (text) pixels, and uncertain pixels. Foreground pixel (darker) usually has a positive value of contrast measure, and most background pixels (lighter) have negative, zero or small positive values. A larger window size yields a better detection of thicker lines patterns while it is difficult to separate very close lines.

Intuitively, this tends to separate the ink from the bottom due to the divergence of the gradient of Markov measurement. Therefore, it will be positive intensity valleys (ink) and negative current peaks or plateaus (bottom). The image contrast is evaluated by the following equation

$$
p_{c}(i, j)=\frac{p_{c, \max }(i, j)-p_{c, \min }(i, j)}{p_{c, \max }(i, j)+p_{c, \min }(i, j)+\epsilon} \ldots \ldots(1)
$$

Where $P c, \max (i, j)$ and $P c, m i n(x ; y)$ refer to the maximum and the minimum image intensities within a local neighborhood window. The term $\epsilon$ is a positive but infinitely small number to avoid dividing zero problems.

Threshold Calculation: Markov random field, apply statically calculate energy function for threshold calculation as show in equation 2 .

$$
c(l)=\sum_{p \in v} c_{1}\left(l_{p}\right)+\beta \sum_{(p . q) \in e} c_{2}\left(l_{p}, l_{q}\right)
$$

Where e (C) tends to maximum likelihood Contrast refer to maximum probability of pixel $\mathrm{p}$ to assigned to label $\mathrm{C}$, and $\mathrm{C} 2(\mathrm{x} ; \mathrm{y})$ refers to the prior Contrast that represents the probability of adjacent pixels p; q are assigned to label lp; lq, respectively.

The Markov random field works for categories. Each pixel indexed by $(i, j)$ is as background pixels, document foreground (text) pixels, and uncertain pixels, $\mathrm{Cij} \in\{0,1\}$.

Contrast reflects the specific labeling fidelity compare to the current data, and corresponding terms for regularity of solution. The contrast includes a $\mathrm{C}^{0} \mathrm{ij}$ or $\mathrm{C}^{1} \mathrm{ij}$, and irregular costs $\operatorname{Ir}^{\mathrm{h}} \mathrm{ij}$ and $\operatorname{Ir}^{\mathrm{v}} \mathrm{ij}$ for every pixel whose label respectively differ from its neighbor on both axis(horizontally and vertically). 


$$
\begin{aligned}
T(c)=\sum_{i=0}^{m} \sum_{j=0}^{n}[\operatorname{Coij}(1-e i j)+C 1 i j e i j] & +\sum_{i=0}^{m} \sum_{j=0}^{n-1} \operatorname{Ir}(e i j \neq e i, j+1) \ldots \\
& +\sum_{i=0}^{m-1} \sum_{j=0}^{n} \operatorname{Irij}(e i j \neq e i+1, j)
\end{aligned}
$$

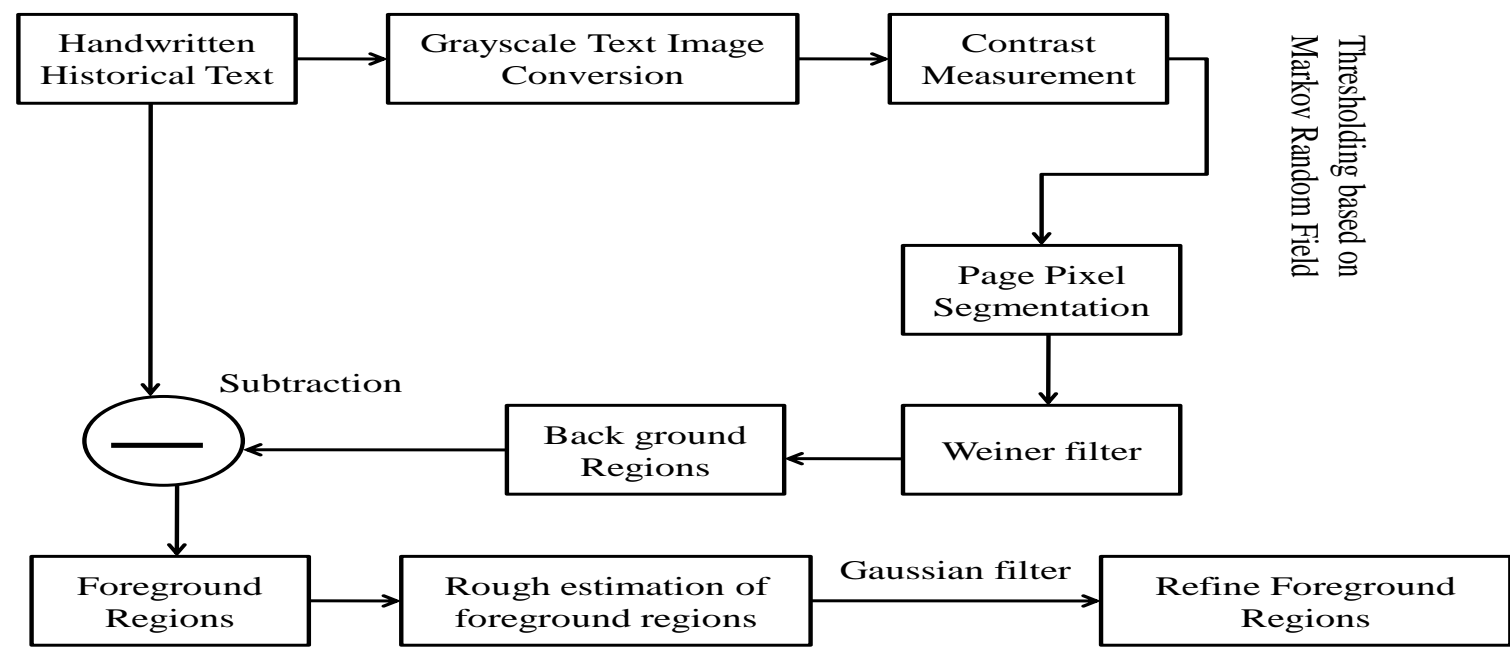

Fig 2: Proposed framework for binarization of historical handwritten text

Weiner filter: - Wiener filter is the MSE-optimal stationary linear filter for images degraded by additive noise and blurring. Calculation of the Wiener filter requires the assumption that the signal and noise processes are secondorder stationary. Proposed scheme use statical threshold calculated by using Markov model to filter out background image as noise. As show in equation 3.

$$
W_{f}(i, j)=\frac{t_{\text {image }} * c_{\text {foreground }}}{\left|t_{\text {image }}\right|^{2} * c_{\text {foreground }}+c_{\text {background }}}
$$

However, if the image contains non-uniform background or too much noise, the contrast of the image may contain several peaks. Using a single threshold value to binaries the entire image would not produce a good binary image.

Rough estimation of foreground regions:- For rough estimation of fore ground regions, proposed framework operator for image pixels with Gaussian filter, it smoothes the image and reduces noise, however, the result of smoothing image causes the fuzzy edge.

Gaussian smoothing is very effective for removing Gaussian noise. The weights give higher significance to pixels near the edge (reduces edge blurring). They are rotationally symmetric, linear low pass filters with higher computational efficiency. The degree of smoothing is controlled by $\sigma$ (larger $\sigma$ for more intensive smoothing). The weights are computed according to a Gaussian function:

$$
G(i, j)=c \cdot e^{\frac{i^{2}+j^{2}}{2 \sigma^{2}}}
$$

\section{ENVIORNMENT SETUP AND RESULT ANALYSIS}

The proposed concept has been implemented in MATLAB due to its large amount of features. In this simulation the handwritten data set of DIBCO13-handwritten has been used. This data set also provides the images which should be a final result. In this work we have calculate the PSNR between the proposed result and the resulting image of the data set. Here it seems to variation in the PSNR value. The proposed approach gives the batter results.

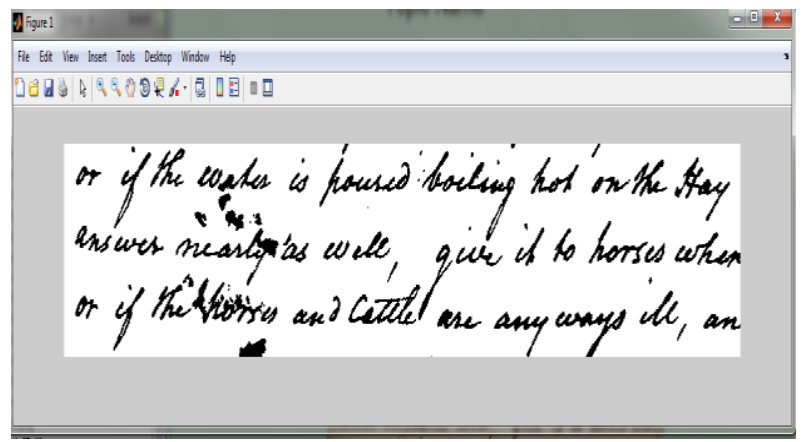

Fig 3: Gray scale conversion on input handwriting image.

After measure contrast measurement and evaluating threshold wiener filter, it filters out background image and resultant foreground image is show in figure 


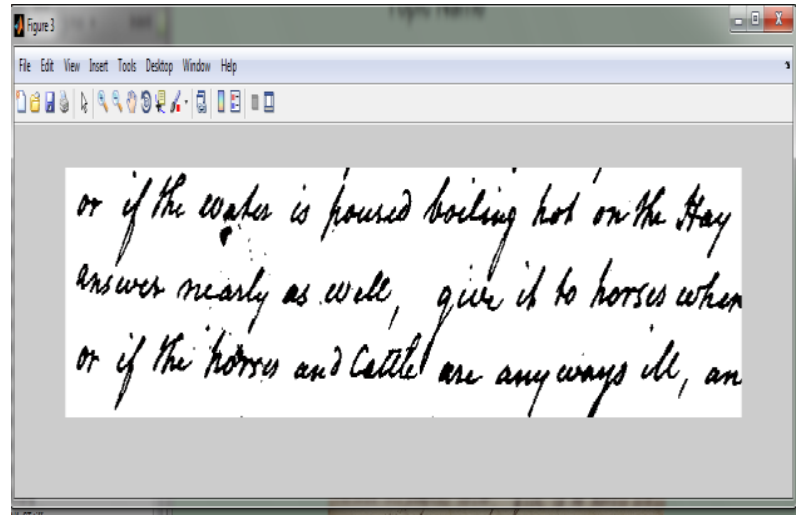

Fig 4: Rough Foreground Image.

Subsequently after filter rough foreground image, rough estimation of fore ground regions, proposed framework operator for image pixels with Gaussian filter, it smoothes the image and reduces noise, however, the result of smoothing image causes the fuzzy edge. Refine foreground image show in figure 5 .

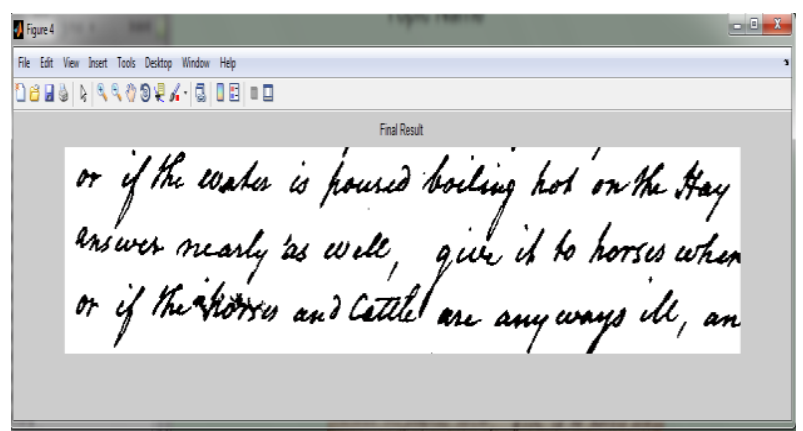

Fig 5: Output handwriting image provided by proposed frame work.

The figure 5 shows the actual output which is provided by the data set DIBCO13-handwritten. Now, to compare the results, we calculate the PSNR values of different handwritten documents from the dataset using both these edge detection algorithms separately.

Below is the Table provided with the PSNR values proposed Contrast MRF and existing robust scheme. Column 2 represents the values of Contrast MRF and column 3 represents the values of robust scheme.

The proposal is that the higher the PSNR, the better degraded image has been reconstructed to match the original image and the better the reconstructive algorithm. This would occur because we wish to minimize the MSE (mean squared error) between images with respect the maximum signal value of the image.

The DIBCO13 contest used some additional primary criteria to describe the quality of the image. Of these, the peak signalto-noise ratio (PSNR) correlates strongly with the quality of image. If $\mathrm{G}$ is the ground truth binary image and $\mathrm{B}$ is the binarization, then

$$
\text { PSNR }=-10 \log ((\Delta \mathrm{G}))
$$

The mean squared error for our practical purposes allows us to compare the "true" pixel values of our original image to our degraded image. Now, from the given table, it can be easily said that the proposed algorithm works best with the MRF algorithm.

Table 1: Comparison Table of PSNR Value

\begin{tabular}{|c|c|c|c|}
\hline \multirow{2}{*}{$\begin{array}{c}\text { Handwriting } \\
\text { Image }\end{array}$} & $\begin{array}{c}\text { Contrast } \\
\text { MRF }\end{array}$ & $\begin{array}{c}\text { Robust } \\
\text { Scheme }\end{array}$ & $\begin{array}{c}\text { Percentage } \\
\text { Improvement }\end{array}$ \\
\cline { 2 - 4 } & \multicolumn{3}{|c|}{ PSNR } \\
\hline 1 & 46.9926 & 41.31 & 12.09254223 \\
\hline 2 & 39.89 & 34.9 & 12.50940085 \\
\hline 3 & 63.09 & 51.7 & 18.05357426 \\
\hline 4 & 75.03 & 69.36 & 7.556977209 \\
\hline 5 & 82.99 & 78.05 & 5.952524401 \\
\hline Average & $\mathbf{6 1 . 5 9 8 5 2}$ & $\mathbf{5 5 . 0 6 4}$ & $\mathbf{1 0 . 6 0 8 2 4 1 8 9}$ \\
\hline
\end{tabular}

As show in table PSNR ratio of proposed contrast MRF is significantly larger than robust scheme. Average percentile improvement is nearly $10.06 \%$. 


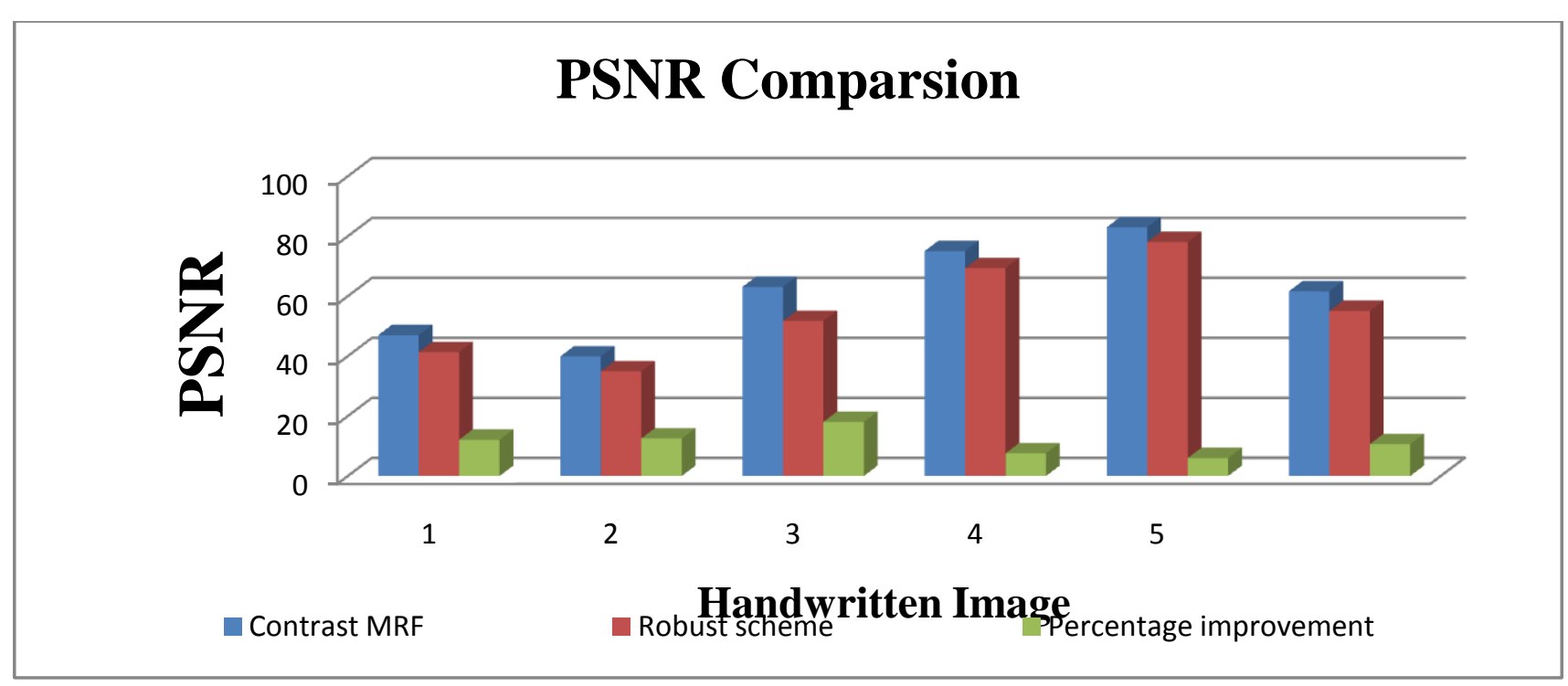

Fig 6: Comparison Graph

\section{CONCLUSION}

Proposed binarization framework inspired by Contrast Markov random field model. Framework consist color to gray conversion, contrast measurement, pixel segmentation based back ground elimination and refine fore- ground image by filter out rough foreground image. With that contrast, the binarization inclined to conform to the contours of intensity while smoothing unevenness resulting because of noise sources. It then introduces a method which calculates the markov of the image intensity to distinguish between the inks from the background. Practically, this approach seem effective, because it gives best location precise ink background transition intensity differences which tend to be large everywhere after a few pixels, so that it becomes very easy to select the place. Markov random field is specially designed for fast classification of objects into two classes. It has successfully been used for noising of images. Proposed method was evaluated on the DIBCO'13 dataset with promising results. The work done in this thesis has overcome the drawbacks of detecting the distorted edges by using edge detection. This framework suggest to used luminous intensity of pixel and try to overcome the problem of appearance of a single document that can vary greatly depending on factors such as viewing angle, shadow. In future try to implement an approach that can predict damage edge of character.

\section{REFERENCES}

[1] Reza Farrahi Moghaddamn, Mohamed Cheriet "AdOtsu: An adaptive and parameter less generalization of Otsu's method for document image binarization", in Elsevier transaction of Pattern Recognition, pg no: 2419-2431, 2012.

[2] B. Gatos, K. Ntirogiannis, I. Pratikakis, "Document image binarization contest (DIBCO 2009)", in International Conference on Document Analysis and Recognition, pg no: 1375-1382, 2009.

[3] Pratikakis, I., Gatos, B., Ntirogiannis, K., "Document image binarization contest (DIBCO 2011)", in International Conference on Document Analysis and Recognition, pg no: 1506-1510, 2011.
[4] Bolan Su, Shijian Lu and Chew Lim Tan, “A Learning Framework for Degraded Document Image Binarization using Markov Random Field" in 21st International Conference on Pattern Recognition (ICPR 2012)November 11-15, IEEE-2012. Tsukuba, Japan

[5] Bolan Su, Shijian Lu, And Chew Lim Tan "Robust Document Image Binarization Technique For Degraded Document Images" in IEEE Transactions On Image Processing, Vol. 22, No. 4, April 2013

[6] David Hebert, Stephane Nicolas and Thierry Paquet "Discrete CRF based combination framework for document image binarization" in 12th International Conference on Document Analysis and Recognition, IEEE-2013

[7] Karthika M Ajay James "A Proposed Method For Document Image Binarization Based on Bit Plane Slicing" in International Conference on Advances in Engineering \&Technology Research (ICAETR - 2014), August 01-02,IEEE-2014

[8] M. Sezgin, B. Sankur, "Survey over image thresholding techniques and quantitative performance evaluation", Journal of Electronic Imaging 13 (1), 146-168, 2004.

[9] R. Farrahi Moghaddam, M. Cheriet, "A multi-scale framework for adaptive binarization of degraded document images", Pattern Recognition 43 (6), 21862198,2010

[10] B. Gatos, I. Pratikakis, S.J. Perantonis, "Adaptive degraded document image Binarization", Pattern Recognition 39 (3) 317-327, 2006.

[11] B. Gatos, K. Ntirogiannis, I. Pratikakis, DIBCO 2009: document image binarization contest, International Journal on Document Analysis and Recognition, 1-10, 2010 . 
[12] J. Fabrizio, B. Marcotegui, M. Cord, "Text segmentation in natural scenes using toggle-mapping”, ICIP'09, pp. 2373-2376, 2009.

[13] B. Gatos, K. Ntirogiannis, I. Pratikakis, ICDAR 2009 document image binarization contest (DIBCO 2009), in: ICDAR'09, pp. 1375-1382, 2009.
[14] R. Hedjam, R. Farrahi Moghaddam, M. Cheriet, "A spatially adaptive statistical method for the binarization of historical manuscripts and degraded document images", Pattern Recognition 44 (9) 2184-2196, 2011.

[15] B. Su, S. Lu, C.L. Tan, "A self-training learning document binarization frame work", ICPR'10, pp. 3187 3190, 2010. 